\title{
Situation und Perspektiven der Filmbildung
}

\author{
Vortrag auf dem 3. Bundeskongress der Kommunalen Kinos, Stuttgart, 11.5.07 \\ Entwicklung in den letzten Jahren
}

Filmerziehung bzw. Filmbildung hat in Deutschland eine lange Tradition. Sie reicht von eher bewahrpädagogischen, normativ orientierten Ansätzen, die in der Nachkriegszeit dominierten, über filmbezogene Ansätze, die über Geschichte, Theorie und Ästhetik des Films informieren und dabei auch die Rezipientenperspektive berücksichtigen, bis hin zu Ansätzen, die den Schritt von der Filmrezeption zur Eigenproduktion mit Film/Video machen (Kinder und Jugendliche erstellen eigene Videofilme). Als Einrichtungen, die langjährig in der Filmbildung in Deutschland aktiv sind, sind im Jugendbereich u.a. zu nennen: das Institut für Medienpädagogik in Theorie und Praxis / Jugend, Film, Fernsehen (JFF München) als älteste Einrichtung der Filmbildung und der Medienpädagogik in Deutschland; das Kinder- und Jugendfilmzentrum Deutschland (mit Sitz in Remscheid); der Bundsverband Jugend und Film (Frankfurt/Main). Hinzu kommen zahlreiche regionale und lokale Einrichtungen wie z. B. Landesmedienzentren, kirchliche Film- und Medienstellen, unabhängige Initiativen und - nicht zuletzt - Kommunale Kinos.

Vor etwa vier Jahren erfolgte der Startschuss zu VisionKino, einer Initiative des Beauftragten der Bundesregierung für Kultur und Medien, der Filmförderungsanstalt, der Stiftung Deutsche Kinemathek und der Aktion Kino macht Schule. In diesem Kontext entstanden Überlegungen zu einem Filmkanon, eine Internet-Plattform wurde aufgebaut, Schulkinowochen in mehreren Bundesländern durchgeführt und in Zusammenarbeit mit der Bundeszentrale für politische Bildung (bpb) Filmhefte zur Unterstützung der Filmbildung herausgebracht. Neben den langjährig existierten Filmbildungseinrichtungen und -gruppen entstanden weitere regionale Initiativen, z. B. in Bayern neben dem bereits erwähnten JFF und den regionalen Fachberatern seit kurzem auch die Kompetenzzentren Film als Fortbildungsangebot mit einer Zertifizierung in Kooperation der Hochschule für Fernsehen und Film in München und der Bavaria Film.

Auch in Baden-Württemberg gibt es diverse Aktivitäten im Bereich Filmbildung, z. B. Schulkinowochen in Kooperation von VisionKino, dem Landesmedienzentrum und nahezu 60 Kinos im Lande; verschiedene Aktivitäten des Ev. Medienhauses; die Aktion kinomobil als ein Beispiel mehrerer von der Medienund Filmgesellschaft Baden-Württemberg (MFG) und der Landesanstalt für Kommunikation (LFK) geförderter Projekte. Auch die im Dezember 2005 veranstaltete Tagung film kreativ gehört hierzu oder das Bundesfestival Video, das 2007 und 2008 als Kooperationsprojekt zwischen dem Bundesjugend- und Familienministerium, dem Kinder- und Jugendfilmzentrum Deutschland, der Stadt Ludwigsburg, der PH Ludwigsburg und der Filmakademie Baden-Württemberg in Zusammenarbeit mit weiterer Einrichtungen der regionalen Film- und Medienarbeit stattfindet. Baden-Württemberg ist ein Bundesland, in dem es inzwischen über 20 Filmfestivals gibt. Bei diesen Festivals spielt - ähnlich wie bei Kommunalen Kinos der Bildungsaspekt eine wachsende Bedeutung. Dass der 3. Bundeskongress der Kommunalen Kinos hier in Stuttgart stattfindet, drückt auch das Engagement Kommunaler Kinos in Baden-Württemberg aus, die seit 30 Jahren vom Land finanziell gefördert werden.

Es gab und gibt aber auch eine kritische Diskussion in den letzten Jahren. Diese betrifft vor allem das Verständnis von Filmbildung und die damit verbundenen Ziele und Arbeitsweisen. So zeigte sich, dass bisherige Initiativen zur Filmbildung teilweise noch sehr einseitig an cineastischen Interessen ausgerichtet sind und zu wenig pädagogische Kontexte und schülerbezogene Arbeitsformen berücksichtigen. Wer z. B. die Filmwelten von Hauptschüler/innen erreichen will, kann nicht den Schwerpunkt auf einen Klassikerkanon von Eisenstein bis Wenders legen. Lehrer/innen benötigen zwar ein filmhistorisches und filmanalytisches Grundwissen, aber dieses Wissen bleibt abgehoben, wenn es nicht mit konkreten Überlegungen für eine adressatenbezogene Filmbildung verknüpft ist. Die folgenden fünf Thesen sprechen einige dieser kritischen Punkte an. Vorweg möchte ich betonen, dass ich seit etwa 25 Jahren in der Film- und Medienbildung engagiert bin. Die Anfänge reichen zurück zu den Odenwälder Jugendfilmtagen, die ich Mitte/Ende der 1980er Jahre im südhessischen Odenwaldkreis durchführte, und gehen bis hin zu aktuellen Filmseminaren im Rahmen der Pädagogik-Ausbildung sowie der Entwicklung eines neuen BachelorStudiengangs Kultur- und Medienbildung an der PH Ludwigsburg. 


\section{These 1}

Filmbildung sollte sich nicht nur auf Kinofilme, sondern auf audiovisuelle Medienangebote insgesamt beziehen.

Für die Diskussion über Perspektiven der Filmbildung ist es zunächst nötig, sich darüber zu verständigen, was unter Film und Filmbildung zu verstehen ist. Nach meiner Auffassung sollten Filme weder auf Kinofilme noch auf thematisch und didaktisch nutzbare Unterrichtsmittel beschränkt werden. Die Bandbreite reicht von Spielfilmen, Dokumentarfilmen, über experimentelle Filme, Werbefilme bis hin zu Medienformaten im Fernsehen. Kurzum: Es geht um Bewegtbilder, die in verschiedenen medialen Speicher- und Distributionskontexten verfügbar sind, die man im Kino, im Fernsehen, auf dem Computer / im Internet, aber auch auf Handydisplays und Leinwänden im öffentlichen Raum findet. Filmbildung bezieht sich also nicht nur auf Kinofilme, sondern auf audiovisuelle Medienangebote insgesamt, deren Nutzung und auch auf die Eigenproduktion von Filmen in unterschiedlichen Kontexten.

Zur Dimension der audiovisuellen Angebote gehören vor allem die filmästhetischen Ausdrucksformen (auch in ihrer historischen Entwicklung), die unterschiedlichen Genres, Formate und Dramaturgien, die filmbezogenen Analysekonzepte, die historischen Entwicklungsphasen der Filmproduktion und distribution (incl. technischer, ökonomischer und rechtlicher Aspekte).

Die Dimension Filmnutzung (Filmrezeption) umfasst vor allem die subjektiven Filmpräferenzen (genrespezifisch, thematisch, ästhetisch), die Aneignungs- und Verarbeitungsformen (kognitiv, emotional, sozial-ästhetisch), die erworbenen Filmkompetenzen im Hinblick auf Auswahl, Verstehen, Analyse und Bewertung audiovisueller Angebote - jeweils in Verbindung mit persönlichen Themen, Nutzungsbedürfnissen und Geschmackspräferenzen.

Schließlich die Dimension der Eigenproduktion von Filmen: diese Dimension meint den Schritt von der Rezeption zur Produktion eigener audiovisueller Beiträge für verschiedene Verwendungszwecke und die Reflexion der dabei gemachten Erfahrungen in filmästhetischer, thematischer und sozial-kommunikativer Hinsicht. Dies sind aus meiner Sicht die zentralen Gegenstandsbereiche von Filmbildung.

Beim bisherigen Konzept von VisionKino liegt eine Verengung auf den Bereich Kinofilm vor. Dies hat mehrere Probleme zur Folge. Um nur zwei gravierende Punkte zu nennen:

- Filmkulturen sind nicht auf Kinofilme und den Ort Kino beschränkt - siehe die Veränderungen bei der Mediennutzung von Jugendlichen in den letzten Jahren, die massenhafte Verwendung von Handys und Internet, das Herunterladen, Produzieren und Versenden von Bewegtbildern, das Anschauen von DVD-Filmen auf großen Flachbildschirmen oder mit Beamern zu Hause.

- Es gibt nicht überall Kinos. Gerade in ländlichen Regionen führt es zu einer strukturellen Benachteiligung, Filmbildungsinitiativen auf den Ort Kino zu begrenzen. Die Filmwirtschaft muss sich in diesem Zusammenhang die Frage gefallen lassen, weshalb sie nicht DVD-Lizenzen für Bildungszwecke frei gibt. Dies ist sicherlich auch europaweit zu klären, aber bislang gibt es seitens der deutschen Filmwirtschaft in dieser Frage keine Bewegung. VisionKino ist eine Initiative, die nur dann eine überzeugende Perspektive hat, wenn sie sich von der Fokussierung auf Kinofilm und den Abspielort Kino löst und ein breiteres Verständnis von Filmbildung entwickelt.

\section{These 2}

Filmbildung findet an verschiedenen Orten und in verschiedenen Kontexten statt.

Um nicht missverstanden zu werden: der Ort Kino ist und bleibt für Filmsehen wichtig. Dazu gehören u.a. eine große Leinwand, ein dunkler Raum, eine sehr gute Akustik. Aber er muss mehr bieten, als ein reiner Abspielort von Filmen zu sein - ich verweise in diesem Zusammenhang auf die guten Vorschläge in der Broschüre zukunft kino des Bundesverbandes kommunale Filmarbeit e.V., auch was die Öffnung für Formen der lokalen Film- und Kulturarbeit betrifft.

Der Ort Kino ist aber nur ein Ort für Filmbildung. Filmbildung ist an verschiedenen Orten sinnvoll, an denen formale und non-formale Bildungsprozesse stattfinden. Non-formale Bildungskontexte sind z. B. die freiwillige Teilnahme an Kinderkinoangeboten oder thematischen Filmreihen im Rahmen von Freizeit- und Bildungsangeboten verschiedener Initiativen und Gruppen. Formale Bildungskontexte, zu denen vor allem die Schulen gehören, sind vor allem durch die verbindliche Teilnahme am Unterricht und an Film- und Video-Kursen (an Nachmittagen) gekennzeichnet, die in pädagogische Settings integriert sind. 


\section{These 3}

Filmbildung umfasst mehr als die Vermittlung von Filmkunst und ist nicht nur filmbezogen.

In der aktuellen Filmbildung lassen sich zwei Grundrichtungen unterscheiden:

a) Von der Filmwissenschaft und der Filmkunst her kommende, mehr filmbezogene Konzepte. Der Schwerpunkt liegt auf der Vermittlung theoretischer, historischer, ästhetischer und analytischer Kenntnisse zum Medium (Kino-)Film. Wesentliche Intention ist die Sensibilisierung der Wahrnehmung und des Geschmacks von Kindern und Jugendlichen für sog. anspruchsvolle Filme. Traditionell werden eher gesprächsorientierte Methoden präferiert, handlungsorientierte Ansätze werden teilweise integriert. Viele Initiativen sind cineastisch orientiert und konzentrieren sich weitgehend auf das Medium Kinofilm. Die Leitfrage ist: „Wie kann Kino als Kunst unterrichtet werden?“ (Bergala 2006)

b) Von der Medienpädagogik und der kulturellen Medienbildung her kommende, mehr subjektbezogene Konzepte. Ausgangspunkt sind die Erfahrungen von Kindern und Jugendlichen im Umgang mit audiovisuellen Medien - Filme werden als wichtiges symbolisches Reservoir für Orientierung, Sinn- und Identitätsbildung betrachtet. Wesentliche Intention ist es, in der Verknüpfung von persönlichkeitsbildenden und filmästhetisch-kulturellen Dimensionen Kompetenzen zu fördern. Eine Mischung aus verbalen und nicht-verbalen Methoden der Filmauswertung sowie handlungsorientierte Ansätze, insbesondere das Erstellen von filmischen Eigenproduktionen, sind der präferierte Ansatz, um filmästhetische Lern- und Reflexionsprozesse zu entwickeln (vgl. verschiedene Beiträge in Niesyto 2006).

Die skizzierten Grundrichtungen sind nicht starr voneinander zu trennen; es gibt Mischformen, die in unterschiedlicher Intensität film- bzw. subjektbezogene Dimensionen oder den Aspekt Eigenproduktion betonen. In außerschulischen, non-formalen Bildungskontexten waren die subjekt- und handlungsorientierten Konzepte schon immer stärker vertreten. Im schulischen Bereich dominierten in der Vergangenheit eher filmbezogene Ansätze und gesprächsorientierte Methoden in Verbindung mit themenspezifischen Aspekten. Die Dominanz von Frontalunterricht und die Einengung durch den 45-Minuten-Rhythmus verhinderten eine stärkere Ausbreitung handlungsorientierter Ansätze. In den letzten Jahren begann sich jedoch an vielen Orten das Blatt zu wenden - immer mehr Schulen öffnen sich für flexiblere Unterrichtszeiten und die Entwicklung zu Ganztagesschulen bietet neue Möglichkeiten zur Integration von außerschulischen Lernorten und Formen aktiv-produktiver Film- und Medienarbeit. Hier sind pädagogischdidaktische Konzepte gefragt, die eine Trennung von Regelunterricht und AG-Wahlangeboten am Nachmittag überwinden und auch in den Regelunterricht Elemente einer aktiv-produktiven Filmarbeit aufnehmen.

Zum Thema Filmkunst: selbstverständlich gibt es Unterschiede in der ästhetischen Qualität von Filmen. Auch ich habe meinen persönlichen Filmgeschmack, mir persönlich stehen Filmästhetiken näher, die z. B. eine dichte symbolische und metaphorische Aussagekraft haben wie z. B. Filme von Kieslowski oder Tarkowski. Wenn ich aber Filmbildung mit Kindern und Jugendlichen mache, kann ich nicht borniert von meinem Filmgeschmack und auch nicht primär von einem filmkünstlerischen Filmkanon ausgehen. Filmbildung setzt - wie alle Bildung - bei den Bedürfnissen, Themen, Erfahrungen derer an, die ich für einen Bildungsprozess motivieren möchte. Viele Kinder und Jugendliche sehen Filme an, die Alain Bergala vermutlich als „schlecht“ einstuft: „Das Leben (...) ist zu kurz, um Zeit und Kraft mit dem Anschauen und Analysieren schlechter Filme zu vergeuden. Vor allem hinterlässt ein schlechter Film, auch wenn man ihn als solchen analysiert, zwangsläufig Spuren und verdirbt den Geschmack, zumal wenn er wiederholt vorgeführt wird und Standbilder betrachtet werden“ (Bergala 2006, S. 40). Diese Haltung ist fatal, läuft sie doch darauf hinaus, Filmerlebnisse von vielen Kindern und Jugendlichen auszugrenzen und abzuwerten. Solche Haltungen verspielen die Chance, an vorhandenen Umgangsweisen und Erfahrungen mit Film anzuknüpfen und in Auseinandersetzung mit diesen Erfahrungen auch die Wahrnehmung für erweiterte Sichtweisen zu öffnen. Beides ist für eine gute Filmbildung notwendig: eine solide Kenntnis im Bereich Filmanalyse, Filmgeschichte, Filmästhetik; aber auch eine Kenntnis der Bedürfnisse und Umgangsweisen, die Kinder und Jugendliche mit Filmen verbinden. Wenn ich z. B. einen Videoclip nehme, kann ich einiges über Filmästhetik, über das synästhetische Zusammenspiel von Musik und Bildern, über assoziative Montageverfahren und auch über Filmgeschichte vermitteln, z. B. dass clipähnliche Montageformen bereits in Filmen wie Ruttmann's Berlin, Sinfonie einer Großstadt entwickelt wurden. Zugestanden - dies ist nicht bei allen Videoclips möglich, aber es müssen nicht Filme sein, die von Videokünstler/innen produziert wurden. 


\section{These 4}

Filmbildung ist mehr als Filmgespräche führen.

Wir kommen damit zur Frage: Wie können wir Filmbildung machen, die die jeweiligen Zielgruppen erreicht? Ich sehe hier vor allem in schulischen Bildungskontexten, aber auch in vielen Beiträgen, die im Umfeld von VisionKino und auch in der Broschüre zukunft kino veröffentlicht sind, eine Verengung von Arbeitsformen auf verbale, kognitiv orientierte Methoden. Notwendig erscheint - gerade im Hinblick auf Kinder und Jugendliche aus bildungsbenachteiligten Milieus - eine stärkere Berücksichtigung alternativer filmpädagogischer Methoden. Es geht darum, konsequent vom filmischen Material auszugehen und konkrete Verarbeitungsmöglichkeiten im Sinne eines anschaulichen Denkens und Reflektierens anzubieten. Beispiele:

- mit Einzelbildern den Verlauf des Films rekonstruieren;

- ein Filmplakat oder eine Collage zum Film gestalten;

- Schlüsselbilder in individuellen Filmstoryboards auswählen (aus digitaler Filmversion ausdrucken und handschriftlich kommentieren);

- auf der Basis kurzer Filmsequenzen Storyboards nachzeichnen und danach eine eigene, kurze Geschichten schreiben und in ein Storyboard transformieren;

- das Prinzip Suspense in filmischen Darstellungen kennen lernen und anhand ausgewählter Filmsequenzen Spannungskurven zeichnen;

- Standbilder erstellen und Filmszenen körpersprachlich erfassen und pantomimisch nachspielen (vgl. Maurer/Niesyto 2006).

Diese alternativen filmpädagogischen Methoden setzen an spezifischen, präsentativ-symbolischen Ausdrucksqualitäten des Mediums Film an und kommen anschaulichen und assoziativen Formen der Weltaneignung bei Kindern und Jugendlichen entgegen.

Zahlreiche Aktivitäten und Projekte zeigen, dass das Erstellen und Öffentlichmachen von Eigenproduktionen mit Video besonders geeignet ist, um filmästhetische, sozial- kommunikative und reflexive Kompetenzen zu entwickeln. Dies trifft insbesondere für Kinder und Jugendliche zu, deren Stärken nicht im Bereich analytisch-kognitiver, abstrahierender Fähigkeiten liegen. Entsprechende Erfahrungswerte sind aus verschiedenen Film- und Videoprojekten bekannt. Viele dieser Erfahrungswerte verdeutlichen die Notwendigkeit, anschauliche und spielerische Arbeitsformen beim Erstellen der Eigenproduktionen stärker zu berücksichtigen, nicht zu komplex vorzugehen, situationsspezifisch Inputs zu geben. Das Erstellen solcher Eigenproduktionen ist nicht nur an außerschulischen Orten, sondern auch im Unterrichtsalltag möglich. So können mit Schüler/innen kleine filmpraktische Übungen gemacht werden, die bezüglich Kameraeinstellung, Perspektiven, Ton-Bild-Korrespondenz etc. Formen des problembezogenen, selbst entdeckenden und kooperativen Lernens eröffnen. Solche Übungen zur aktiven Filmgestaltung sind ein praxisbezogener Einstieg in die „Welt des Films“ und motivieren oft Schüler/innen zu weiteren Eigenproduktionen, die dann z. B. im Rahmen von AGs realisiert werden können.

\section{These 5}

Filmbildung ist im Kontext von Medienbildung verbindlich in allen pädagogischen Studiengängen zu verankern. Filmbildung als eigenes Schulfach ist zu eng gefasst.

Obgleich audiovisuelle Medien in den Lebenswelten von Kindern und Jugendlichen eine zentrale Bedeutung für die Sozialisation haben und diverse Studien vorliegen, die dies belegen, mangelt es nach wie vor an einer verbindlichen Verankerung von Medien- und Filmbildung in den Studien- und Prüfungsordnungen zur Lehrerausbildung und in anderen pädagogischen Studiengängen. Es gibt zwar Aussagen in Stellungnahmen der Kultusministerkonferenz, aber die Situation in den einzelnen Bundesländern und Hochschulen ist sehr unterschiedlich. Die Spannweite reicht von eher marginalen, punktuellen Seminarangeboten über eine deutliche Verstärkung mediendidaktisch orientierter Module in Lehramtsstudiengängen bis hin zur Einrichtung neuer Studiengänge. Selbst an Hochschulen, an denen in verschiedenen Fächern Seminare zu Filmgeschichte, Filmgestaltung und Filmbildung angeboten werden, fehlt oft eine fächerübergreifende Abstimmung der Angebote. 
Bei den Umstellungen bisheriger Lehramts-, Diplom- und Magister-Studiengänge in das Bachelor/Mastersystem ist ein Trend zur Komprimierung und Standardisierung von Ausbildungsinhalten zu beobachten, der nicht etablierte Ausbildungsinhalte wie Filmbildung vom Kanon der Kerncurricula fernhält und weiter marginalisiert. In der zweiten und dritten Phase der Lehrerbildung verschärft sich die Situation noch. Filmbildung ist vielerorts kein Gegenstand von Lehrerfortbildung. Die wenigen Angebote - meine Beobachtungen beziehen sich hier auf Baden-Württemberg - werden von Lehrer/innen unzureichend genutzt. Als Gründe sind u. a. eine Zuordnung von Film zu Freizeit und Unterhaltung, eine Unkenntnis über die generelle Bedeutung von Filmbildung und ein zu geringer Bezug von Fortbildungsangeboten auf die konkrete Situation vor Ort zu nennen. Im Bereich der „Frühen Bildung“, der bildungspolitisch derzeit boomt, entstehen zwar zahlreiche neue Ausbildungsinitiativen; doch auch hier gestaltet sich die Situation recht heterogen. Als ein übergreifender Problembereich lässt sich eine Art filmkulturelle Kluft zwischen den Filmpräferenzen vieler Pädagog/innen und denen vieler Jugendlicher beschreiben. Man kann von unterschiedlichen medialen Symbolmilieus oder Habitusformen sprechen, die sich bei Pädagogen u. a. in Nichtkenntnis, Stereotypen, pauschalen Abwertungen gegenüber populären Filmangeboten und deren Rezeption ausdrücken.

Aus dem Umfeld der Initiative VisionKino, insbesondere von Filmschaffenden, kommt immer wieder die Forderung nach einem eigenen Schulfach „Film“. Film sei das Leitmedium der letzten hundert Jahre und müsse „mindestens denselben Stellenwert wie die Literatur im Unterricht haben“ so z. B. der Regisseur Florian Henckel von Donnersmarck auf dem 2. Berliner Kongress von VisionKino im Oktober 2006. So richtig der Hinweis auf die Filmvergessenheit der Pädagogik ist, so problematisch und unrealistisch sind doch Forderungen, die gleichsam in einem ,Hau-Ruck-Verfahren’ eine bundeszentral angeordnete Einführung eines Schulfachs „Film“ - etwa nach dem Vorbild Frankreichs - anstreben. Zum einen kann es nicht darum gehen, isoliert Film und Filmbildung in den Lehrplänen an Hochschulen und in den Bildungsplänen an Schulen zu verankern. Filmkompetenz und Filmbildung sind ein integrierter Teil von Medienkompetenz und Medienbildung, die sich nicht nur auf audiovisuelle Medien beziehen. In einer Situation, in der nicht einmal Medienbildung als verbindlicher, prüfungsrelevanter Teil für alle Studierenden in den Studien- und Prüfungsordnungen für das Fach Erziehungswissenschaft verankert ist, scheint es mir völlig unrealistisch, isoliert Filmbildung als eigenständiges Fach zu etablieren. Hinzu kommt, dass wir in Deutschland eine föderale Struktur haben und bildungspolitische Fragen vor allem Ländersache sind.

Bundesweite Empfehlungen, Kongresse, Modellprojekte können hilfreich sein und es ist sinnvoll, auf Bundesebene über Standards für Filmbildung nachzudenken. Letztlich sind jedoch Personen und Institutionen in den einzelnen Regionen und Ländern gefordert, konkrete Schritte in Richtung einer besseren Verankerung der Filmbildung in den Lehr- und Bildungsplänen zu machen. Hier gibt es in den letzten Jahren durchaus Fortschritte, wie z. B. das bayerische Pilotprojekt „Entwicklung und Erprobung eines Curriculums zur Ausbildung von Lehrkräften im Bereich Schulfilm mit abschließender Zertifizierung“ zeigt. Für die erste Phase der Lehrerbildung an Hochschulen ist es wichtig, dass es sowohl auf der Ebene der einzelnen Hochschulen als auch auf Landesebene (Fachschaften) zu einem institutionalisierten Austausch zwischen den Kolleginnen und Kollegen kommt, die an Filmbildung interessiert sind, um Ausbildungsinhalte besser untereinander abzustimmen und Vorschläge für künftige Novellierungen von Studien- und Prüfungsordnungen zu erarbeiten. Dies betrifft insbesondere die Fächer Erziehungswissenschaft, Deutsch, Kunst, Musik; weitere Fächer sollten einbezogen werden.

Seminarangebote zu Grundlagen der Filmgestaltung, der Filmanalyse, der Filmtheorie, der Filmgeschichte sind durchaus vorhanden. Es gibt inzwischen brauchbare Materialien, die auch im Rahmen von Selbststudienanteilen gut einsetzbar sind, z. B. zur Filmanalyse die DVD von Rüdiger Steinmetz (2005) oder die DVD zum Buch Jugend:Film:Kultur, das ich 2006 zusammen mit Werner Barg von Deutschen Filmund Fernsehakademie Berlin und Jan Schmolling vom Kinder- und Jugendfilmzentrum Deutschland herausgab (Barg u.a. 2006). Entsprechende Grundkurse sollten fächerübergreifend abgestimmt und angeboten werden, um Basiswissen zu vermitteln. Für die filmbildnerische Arbeit reichen solche Grundkurse jedoch nicht aus. Weitere wichtige Themenfelder für Seminarangebote sind: 
- $\quad$ Auseinandersetzung mit der eigenen Film- und Mediensozialisation, z. B. im Rahmen kleiner medienbiographischer Studien;

- Kennenlernen und Auseinandersetzung mit Filmwelten und Filmpräferenzen von Kindern und Jugendlichen, z. B. im Rahmen von Praktika und Filmprojekten an Schulen;

- Kennenlernen unterschiedlicher Konzepte für eine zielgruppenbezogene Filmbildung, z. B. für eine Filmbildung an Grundschulen, in der Sekundarstufe I und II, für gemeinwesenorientierte Formen der Filmbildung (auch im Kontext von Nachmittagsangeboten);

- Verknüpfungsmöglichkeiten von filmbezogenen und fachbezogenen Fragestellungen, z. B. Seminare zu „Das Lehrer-Schüler-Verhältnis in ausgewählten Spiel- und Dokumentarfilmen“, „Geschichtsbilder in Kinofilmen“ oder „Filmmusik“;

- Verknüpfungsmöglichkeiten von filmbezogenen und fächerübergreifenden Fragestellungen, z. B. Seminare und Praxisprojekte zu „Jugendkulturen in filmischen Eigenproduktionen“ oder „Filme als Brücke zur Welt“.

Erfahrungsgemäß reicht die Vermittlung von Fachkenntnissen an den Hochschulen nicht aus. Damit sich Motivationen und Kompetenzen tiefer und nachhaltiger entwickeln können, sind vor allem praktische Erfahrungen in pädagogischen Handlungsfeldern notwendig. Hierfür ist die Kooperation von Ausbildungseinrichtungen mit Facheinrichtungen der Film- und Medienarbeit, Schulen und Jugendeinrichtungen in der Region zu fördern. Sinnvoll erscheint besonders der gezielte Aufbau einzelner Stützpunktschulen und Praxiseinrichtungen, in denen Lehrer/innen und (medien-)pädagogische Fachkräfte einen kontinuierlichen Schwerpunkt auf Filmbildung legen. Für die schrittweise Realisierung solcher Vorhaben sind gemeinsame, projektbezogene Aktivitäten besonders geeignet - Fachleute aus verschiedenen Bereichen arbeiten zusammen, lernen voneinander, verständigen sich auf gemeinsame Zielsetzungen, bieten Studierenden Möglichkeiten zur praktischen Mitarbeit.

Filmbildung sollte die vielfältigen Dimensionen, die im Medium Film und in Filmkulturen enthalten sind, erheblich stärker für lokalbezogene Aktivitäten nutzen - von kreativen Nachspielaktionen in Kooperation mit Kindergärten und Grundschulen (Kinderkino) über jugendkulturell orientierte Filmreihen und Filmprojekte bis hin zu Formen einer Oral History zur lokalen Filmkultur oder zu Produktionsprojekten, an denen auch professionelle Filmschaffende beteiligt sind. Gleichzeitig ist darauf zu achten, die Breitenwirksamkeit von Filmbildung zu erhöhen. In diesem Zusammenhang sind Angebote wie Schulfilmwochen, spezielle Wettbewerbe und Festivals weiterzuentwickeln. Hierzu gehört auch der Aufbau eines Info-Pools, der über Filmbildungsfachleute in der Region, Literatur und Materialien zur Filmbildung, Veranstaltungen etc. informiert. Damit komme ich zum abschließenden Teil meines Vortrags, in dem ich auf Chancen der Filmbildung für die Kommunalen Kinos eingehen möchte.

\section{Chancen für die Kommunalen Kinos}

Welche Chancen haben Kommunale Kinos, um nicht zwischen kommerziellen Kinoangeboten und einem sehr spezialisierten Spartenkinoprogramm für Minderheiten zerrieben zu werden? Es bleibt mit Sicherheit eine wichtige Aufgabe Kommunaler Kinos, die Geschichte von Film und Filmkunst im kulturellen Gedächtnis der Menschen lebendig zu halten - gerade in einer Zeit rigoroser Kommerzialisierung des gesamten Film- und Medienbereichs, des Verwischens von Formaten und einer sehr starken Quoten- und Renditeorientierung. Die Frage ist aber, wie diese Aufgabe mit anderen Herausforderungen verknüpft und neue Zielgruppen erreicht werden können. Hierzu möchte ich ein paar Vorschläge machen.

- Stärkere Öffnung für neue Zielgruppen jenseits eines cineastisch orientierten Publikums. Hier bieten sich vor allem Kooperationsprojekte mit verschiedenen Schulen (nicht nur Gymnasien!) im Vormittags- und im Nachmittagsunterricht an, die handlungsorientierte Formen der Filmbildung (siehe These 4) integrieren. Es geht darum, eine Fixierung auf sprachorientierte Methoden der Filmbildung zu überwinden und durch sinnlich-ästhetische Gestaltungsmöglichkeiten Kinder und Jugendliche zum Mitmachen zu motivieren. Wichtig sind auch Initiativen, die Eltern im lokalen Gemeinwesen erreichen, um über Film- und Medienangebote zu informieren und Tipps zur Filmund Medienerziehung ihrer Kinder zu geben. Hierzu gehören auch bessere Informationen über die sog. FSK 12-Filme. Diese Kinofilme, die ab 12 Jahren freigegeben sind, dürfen seit dem 1. April 2003 von Kindern ab 6 Jahren in Begleitung von personensorgeberechtigten Erwachsenen besucht werden. 
- Die „Drehschreibenfunktion“ von Kommunalen Kinos stärker betonen. Unter „Drehscheibenfunktion“ verstehe ich die Möglichkeit, Kommunale Kinos als Begegnungs- und Kommunikationsraum zwischen Filmschaffenden und Zuschauer/innen zu nutzen. Die Filmakademien in Deutschland bieten viele junge Nachwuchsfilmer/innen aus. Gerade dieses Potenzial sollte man seitens der Kommunalen Kinos gezielter ansprechen: Kommunale Kinos als Plattform für Filmpräsentationen von Nachwuchsfilmer/innen und als Ort für Gespräche und Feedbacks. Nachwuchsfilmer/innen brauchen diese Feedbacks, sie möchten wissen, wie ihre Filme beim Publikum ankommen.

- Kommunale Kinos als Teil soziokultureller Bildungsarbeit vor Ort. Bereits jetzt gibt es in vielen Kommunalen Kinos Angebotsstrukturen, die auf der Zusammenarbeit mit unterschiedlichen soziokulturellen Gruppen vor Ort beruhen und entsprechende Filme und Filmreihen anbieten und in manchen Fällen - sogar spezielle Festivals organisieren. Solche öffentlichen Begegnungs- und Kommunikationsräume in sozialen Nahräumen sind gerade in einer Zeit wichtig, in der die gesellschaftliche Individualisierung viele Menschen in virtuelle Kommunikationsräume treibt, die jedoch für lebendige Begegnungen und Austausch im Real-Life keinen Ersatz bieten können. Kommunale Kinos sollten diese Funktion ausbauen und neben filmbezogenen Angeboten auch die Kooperation mit anderen Bereichen wie Theaterspiel, Musik usw. suchen.

- Mitwirkung bei der Entwicklung von Angeboten zur Aus-, Fort- und Weiterbildung. In diesem Feld bieten sich verschiedene Möglichkeiten an, vorhandenes Spezialwissen von Mitarbeiter/innen aus Kommunalen Kinos einzubringen. Ich denke z. B. an eine Mitwirkung bei Fortbildungsveranstaltungen für Lehrer/innen und pädagogische Fachkräften (die z. B. Chancen unterschiedlicher Filmgenres für die Bildungsarbeit herausarbeiten), an Bausteinangebote für Unterricht und Jugendarbeit zu ausgewählten Filmen und Themen oder an die Mitwirkung bei einer längerfristig angelegten Fortbildungsreihe zur Filmbildung (zusammen mit Filmschaffenden, Filmwissenschaftlern, Filmpädagogen).

Die Realisierung dieser Aktivitäten ist ohne eine finanzielle Basis nicht möglich. Wer unter Filmförderung mehr versteht als die finanzielle Förderung bestimmter Filmprojekte für Kino und Fernsehen, der muss erheblich mehr in den Bereich Filmbildung investieren! Länder, Kommunen und entsprechende Facheinrichtungen sind gefordert, hier neue Maßstäbe zu setzen. Qualitätsbezogene Filme brauchen ein Publikum, das filmische Qualität erkennen kann. Filmbildung ist hierfür unerlässlich.

\section{Literaturhinweise}

Barg, Werner / Niesyto, Horst / Schmolling, Jan (Hrsg.): Jugend:Film:Kultur. Grundlagen und Praxishilfen für die Filmbildung. Mit einer DVD. München 2006.

Bergala, Alain: Kino als Kunst. Filmvermittlung an der Schule und anderswo. Marburg 2006.

Bundesverband kommunale Filmarbeit e.V. (Hrsg.): zukunft kino. Frankfurt/Main 2006.

Maurer, Björn / Niesyto, Horst: Filmbildung in Hauptschul- und Migrationsmilieus. In: tv-diskurs, 10. Jhg., Nr. 4 (2006), S. 10-11.

Niesyto, Horst (Hrsg.): film kreativ. Aktuelle Beiträge zur Filmbildung. Reihe Medienpädagogik interdisziplinär (Band 6). München 2006.

Steinmetz, Rüdiger: Filme sehen lernen. Grundlagen der Filmästhetik (DVD mit Begleitbuch). Frankfurt/Main 2005.

Kontakt: niesyto@ph-ludwigsburg.de; www.ph-ludwigsburg.de/medien1 